Calibration of a High Resolution Grating Soft X-ray Spectrometer

E. W. Magee, J. Dunn, G. V. Brown, K. V. Cone, J. Park, F. S. Porter, C. A. Kilbourne, R. L. Kelley, P. Beiersdorfer

August 25, 2010

Review of Scientific Instruments 
This document was prepared as an account of work sponsored by an agency of the United States government. Neither the United States government nor Lawrence Livermore National Security, LLC, nor any of their employees makes any warranty, expressed or implied, or assumes any legal liability or responsibility for the accuracy, completeness, or usefulness of any information, apparatus, product, or process disclosed, or represents that its use would not infringe privately owned rights. Reference herein to any specific commercial product, process, or service by trade name, trademark, manufacturer, or otherwise does not necessarily constitute or imply its endorsement, recommendation, or favoring by the United States government or Lawrence Livermore National Security, LLC. The views and opinions of authors expressed herein do not necessarily state or reflect those of the United States government or Lawrence Livermore National Security, LLC, and shall not be used for advertising or product endorsement purposes. 


\title{
Calibration of a High Resolution Grating Soft X-ray Spectrometer ${ }^{\text {a) }}$
}

\author{
E. W. Magee, ${ }^{1}$ J. Dunn, ${ }^{1, b)}$ G. V. Brown, ${ }^{1}$ K. V. Cone, ${ }^{1,2}$ J. Park, ${ }^{1,2}$ F. S. Porter, ${ }^{3}$ \\ C. A. Kilbourne, ${ }^{3}$ R. L. Kelley, ${ }^{3}$ and P. Beiersdorfer ${ }^{1}$ \\ ${ }^{1}$ Lawrence Livermore National Laboratory, P.O. Box 808, Livermore, California 94550, USA \\ ${ }^{2}$ Department of Applied Sciences, University of California, Davis, California 95616, USA \\ ${ }^{3}$ NASA/Goddard Space Flight Center Greenbelt, MD 20770, USA
}

(Presented XXXXX; received XXXXX; accepted XXXXX; published online XXXXX)

The calibration of the soft $\mathrm{X}$-ray spectral response of a large radius of curvature, high resolution grating spectrometer (HRGS) with a back-illuminated charge-coupled device detector is reported. The instrument is cross-calibrated for the $10-50 \AA$ waveband at the Lawrence Livermore National Laboratory electron beam ion trap (EBIT) x-ray source with the EBIT calorimeter spectrometer (ECS). The HRGS instrument is designed for laser-produced plasma experiments and is important for making high dynamic range measurements of line intensities, line shapes and x-ray sources.

\section{INTRODUCTION}

The development of x-ray spectroscopic instrumentation continues to be important for further understanding of the processes and physics in high temperature and high energy density plasmas. The measurement and correct interpretation of soft x-ray spectra, relative line intensity ratios, continuum slopes, spectral line shapes and source $\mathrm{x}$-ray emissivity, can yield a wealth of information on the plasmas. ${ }^{1-4}$ This invariably requires a methodology for calibrating the instrument sensitivity function over the waveband of interest. For soft x-rays in the $10-500 \AA$ range, grating spectrometers, for example, ${ }^{5}$ are frequently fielded. The calibration can be technically challenging as instruments using grazing incidence mirrors or gratings are susceptible to the contamination of thin hydrocarbon layers where even 10 nanometer coatings can have a significant effect on the reflectivity. Also the use of thin sub-micron filters, which may be affected by oxidation or have non-uniform thickness, for light tightness or signal attenuation require the transmission to be accurately determined. ${ }^{6}$ The detection efficiency of chargecoupled device (CCD) detectors using back-thinned chips can be affected by thin dead layers of silicon and silicon oxide.

We report the calibration of a High-Resolution variablespaced Grating, flat-field Spectrometer (HRGS) ${ }^{8,9}$ with a backthinned CCD detector for the wavelength range of $10-50 \AA$ on the electron beam ion trap (EBIT) $x$-ray source at the Fusion and Astrophysics (FAST) data and instrument calibration facility at the Lawrence Livermore National Laboratory (LLNL). ${ }^{10,11}$ The EBIT calorimeter spectrometer (ECS) ${ }^{12}$ was used to crosscalibrate the grating instrument on EBIT-I by recording the soft $\mathrm{x}$-ray emission simultaneously. We show an example of the recorded spectra from both instruments. The HRGS efficiency, $\varepsilon$, defined here as grating reflectivity $\times$ quantum detection efficiency product, is measured to be $1-1.6 \%$ for the $15-30 \AA$ wavelength range by taking into account the solid angles, source emission volume and filters used in the calibration run for the two instruments. We describe the methodology involved in the calibration, some of the limitations as well as areas that will be pursued in the future.

\section{EXPERIMENTAL DESCRIPTION}

The HRGS soft x-ray spectrometer consists of a 2400 line/mm variable spaced grating of dimensions $5 \times 10 \mathrm{~cm}^{2}$ with a radius of curvature of $R=44.3 \mathrm{~m}$ to disperse the incident spectrum in a flat-field detection plane. ${ }^{8}$ The HRGS spectrometer has recorded spectra on EBIT-I and laser-produced plasma x-ray sources. ${ }^{8,9}$ The calibration of the HRGS instrument was performed at FAST using EBIT-I. Various source gases including $\mathrm{CO}_{2}, \mathrm{~N}_{2}$, and $\mathrm{Ne}$ were injected into the trap at a nominal pressure of $10^{-6}$ Torr. These generated $\mathrm{K}$-shell soft $\mathrm{X}$-ray emission lines of interest in the $10-50 \AA$ wavelength range mostly from the $\mathrm{H}$ like and He-like charge states. The continuous electron beam, nominally of $60 \mu \mathrm{m}$ beam diameter, was held at a constant energy and ionized and excited the gas in the trap to the K-shell ion stage. The ion trap region and $\mathrm{x}$-ray emission region was nominally $2 \mathrm{~cm}$ in length and defined axially by the voltages applied to the electrodes in the drift section. The ion trap voltage was pulsed to periodically empty the trap and duration time was defined by the cycle time. The electron beam energy and current together with the cycle time were optimized to maximize the $\mathrm{x}$ ray source emission: $3.08 \mathrm{keV}, 132 \mathrm{~mA}$ and $49 \mathrm{~ms}$ was used for the $\mathrm{CO}_{2}$ and $\mathrm{N}_{2}$ gases while the $\mathrm{Ne}$ gas was run at $5.0 \mathrm{keV}, 76$ $\mathrm{mA}$ and $105 \mathrm{~ms}$.

The grating spectrometer and the ECS, separated by $90^{\circ}$, viewed the $x$-ray source in the horizontal plane. The grating spectrometer was run without a slit to maximize the $\mathrm{x}$-ray signal at the detector and was orientated so that the source was parallel to the groove direction. The source size and $\mathrm{x}$-ray emission region therefore determined the instrument spectral resolution. A series of baffles between the source and the grating were set to illuminate the grating and reduce scatter inside the spectrometer. No light tight filter was required. The $\mathrm{x}$-ray source to grating center was measured to be $194.6 \mathrm{~cm}$. The spectrometer grating to detector distance was adjusted to achieve the best focus of the spectral lines and was approximately $150 \mathrm{~cm}$ depending on the wavelength setting. The grating surface was inclined at an angle of $2^{\circ}$ to the source. A Princeton Instruments, liquid-Nitrogencooled (operated at $-110^{\circ} \mathrm{C}$ ) back-thinned $\mathrm{CCD}$ detector was placed at the flat-field detection plane. The CCD contained a

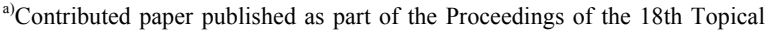
Conference on High-Temperature Plasma Diagnostics, Wildwood, New Jersey, May, 2010.

b)Electonic mail: dunn6@1lnl.gov. 
$1340 \times 1300$ pixel array with each pixel having a $20 \times 20 \mu \mathrm{m}^{2}$ dimension. The $x$-ray calibration runs typically lasted 60 minutes per exposure to increase the total number of detected x-ray photons. The CCD signal was digitized by a low noise 16-bit 50 $\mathrm{kHz}$ analog to digital convertor (ADC) at the high gain setting, $g$, of 1.07 elec./ADC count. The recorded 2-dimensional CCD images were analyzed as follows: A background image was numerically subtracted and a small tilt angle of $0.15^{\circ}$ was applied to the spectral lines to align with the CCD array. Cosmic ray events were removed in 2 stages by pixel value analysis threshold techniques to set hot pixels to background values followed by nearest pixel value comparisons. This was found to effectively remove most of the cosmic rays with a very small effect of reducing the peak $\mathrm{x}$-ray emission lines by less than $2 \%$. The signal was then averaged over the full height of the line (1290 pixels) to generate a spectrum. The mainly $\mathrm{H}$-like and He-like ion emission lines were identified and a wavelength dispersion applied using the reference values tabulated in Kelly. ${ }^{13}$ The measured line signal, $S$, in ADC counts was converted to detected photons, $N$, using the relation $N=(S g \omega) / E$ where $g$ is the above ADC gain, $\omega$ is the Si work function of $3.65 \mathrm{eV} / \mathrm{elec}$. and $E$ is the photon energy.

The EBIT calorimeter spectrometer is a solid-state energy dispersive device first developed at NASA's Goddard Space Flight Center in $1984 .^{12}$ The present ECS consists of a $6 \times 6$ array of $\mathrm{HgTe}$ pixels cryogenically-cooled to $50 \mathrm{mK}$ using an adiabatic de-magnetization refrigerator in a liquid ${ }^{3} \mathrm{He} /{ }^{4} \mathrm{He}$ bath. The array consists of $624 \times 624 \mu \mathrm{m}^{2} \times 8 \mu \mathrm{m}$ thick pixels for mid-energy 0.1 $-10 \mathrm{keV}$ photons interspersed with $624 \times 500 \mu \mathrm{m}^{2} \times 100 \mu \mathrm{m}$ thick pixels for improved high energy $0.5-100 \mathrm{keV}$ photon detection. HgTe is chosen as the detector material because of high x-ray absorption and low heat capacity. X-ray absorption is $100 \%$ for photon energy below $4 \mathrm{keV}$. The energy resolution when operated at the $50 \mathrm{mK}$ cryogenic temperature is $\Delta E \sim 5 \mathrm{eV}$ at $6 \mathrm{keV}$ and $\Delta E \sim 25 \mathrm{eV}$ at $60 \mathrm{keV}$ photon energy. Thermal isolation of the calorimeter is achieved with 4 thin foils of aluminized polyimide $\left(\mathrm{C}_{22} \mathrm{H}_{10} \mathrm{~N}_{2} \mathrm{O}_{5}\right)$ with a total thickness of $147.0 \mathrm{~nm} \mathrm{Al} / 238.6 \mathrm{~nm}$ polyimide. The absorption of this filter set has to be corrected in the calibration for low energy photons under study here. A fifth filter of $21 \mathrm{~nm} \mathrm{Al} / 1030 \mathrm{~nm}$ polyimide was added to attenuate the X-ray signal further onto the ECS to minimize signal pile-up. In the data analysis the signal from 16 of the mid-energy pixels was combined to give high photon statistics. The array was positioned $95.17 \mathrm{~cm}$ from the source and observed the center $1.65 \mathrm{~cm}$ length of the cylindrical ion beam.

\section{RESULTS}

A sub-set of the data is reported in this paper to illustrate the cross-calibration technique. The HRGS results have been converted from wavelength to energy scale to directly compare the spectra from the two instruments as shown in Fig. 1 for nitrogen gas ionized by the electron beam. The spectra from both instruments are plotted as total detected photons versus energy. The data were recorded for 60 minutes. The energy range of 400 - $600 \mathrm{eV}$ represents the full spectral coverage of the grating instrument at one setting limited by the CCD detector size. The strong $n=2-1$ and $3-1$ lines are labeled as well as some of the higher order He-like ion series. It can be seen that the HRGS spectrum is well resolved. Spectral resolution of $E / \Delta E \sim 1300$ is determined by the EBIT source size and corresponds to $\Delta \mathrm{E}$ of $0.34 \mathrm{eV}$ (FWHM) or equivalent to $3 \mathrm{CCD}$ pixels for the $\mathrm{N} \mathrm{He}-\alpha$ line at $430.7 \mathrm{eV}$. Residual oxygen ions are present in the ion trap and this accounts for the weak He- $\alpha$ line at $574 \mathrm{eV}$. The ECS covers a much larger range (not shown in the figure) and has the advantage of recording photon energies from $100 \mathrm{eV}$ to $100 \mathrm{keV}$ simultaneously. The ECS spectrum (in red) does not resolve the He- $\beta$ line on the low energy side of the stronger Ly- $\alpha$ line at 500 $\mathrm{eV}$ due to the lower energy resolution of $\Delta \mathrm{E} \sim 5 \mathrm{eV}$. It can also be noted that the line intensity ratios for the two instruments change across the spectra. The ECS results as shown are not corrected for the filter response and this strongly attenuates the lower photon energies.

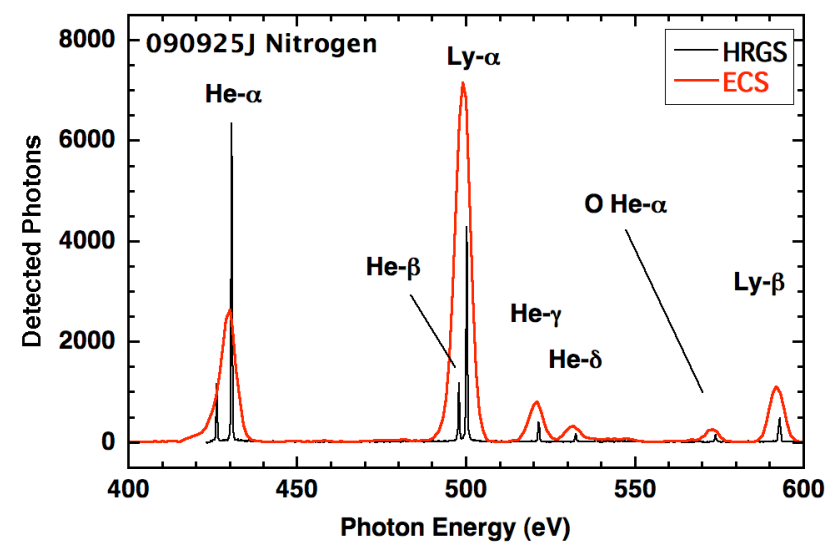

FIG. 1. Soft x-ray nitrogen spectrum recorded from EBIT source showing strong $\mathrm{He}$-like and $\mathrm{H}$-like lines showing detected photons versus photon energy. The ECS spectrum (in red) is shown while the grating spectrum is in black. (Color online)

The strongest $\mathrm{x}$-ray lines for nitrogen and oxygen were analyzed for the two instruments to calibrate the HRGS efficiency, $\varepsilon$, defined here as grating reflectivity $\times$ quantum detection efficiency product for $15-30 \AA$. For this study it was not possible to separate the grating reflectivity from the CCD quantum detection response. The geometry of each spectrometer was taken into consideration including the solid angle subtended to the source, the emission volume observed by the detectors as well as any filter corrections. The ECS solid angle was $6.9 \times 10^{-6}$ steradians. The HRGS instrument solid angle was determined by the effective grating height tilted towards the source in the plane of dispersion (1.79 mrad) and the angle subtended by the detector orthogonal to the plane of dispersion $(7.62 \mathrm{mrad})$. This latter number was measured separately in a laser plasma experiment by placing an aperture in front of the grating close to the $\mathrm{x}$-ray point source to determine (a) the fraction of the grating illuminated by the $\mathrm{x}$-rays reaching the detection plane and (b) the degree of focusing along the spectral line height. It was found that there was no focusing of $\mathrm{x}$-rays in the plane orthogonal to the dispersion. The HRGS solid angle was $1.37 \times 10^{-5}$ steradians or twice the ECS instrument for the EBIT calibration run. Very small corrections $(<5 \%)$ were made for the observed emission volumes of the two instruments. For some spectral lines the ECS is unable to resolve close lines, as shown in Fig. 1, in which case the line intensity for the grating spectrometer is integrated over the full width observed by the ECS instrument.

Figure 2 shows the grating efficiency as a function of the $\mathrm{x}$ ray wavelength in the $15-30 \AA$ range. Values of $1-1.6 \%$ are measured where the absolute response is gradually decreasing at shorter wavelengths as a result of the falling grating efficiency. The error bars of $\sim \pm 15 \%$ are largely determined by a number of factors but are not limited by the photon statistics in individual 
lines. X-ray lines recorded in the grating spectrometer are determined to have wings due to the grating response function from surface roughness effects and are readily observed in the stronger lines. Precise fitting of the line shape is required. Secondly, background photons or low level noise that extend away from the lines that are not wings or part of the line (and may not be in the ECS data) are included in the signal as a result of the wider energy range of the ECS. This becomes important for weaker lines recorded in the calibration. These effects have to be addressed carefully and will require further detailed analysis. Studying high-resolution ECS events could help to determine the overall contribution in the latter case. The third contribution comes from the transmission response of the thin filter set used in front of the ECS. The filter transmission will be calibrated in the near future. It can be noted here that previous filter measurements conducted on the ECS have shown that the filter samples and thicknesses supplied by Luxél have been very precise. ${ }^{6,14}$

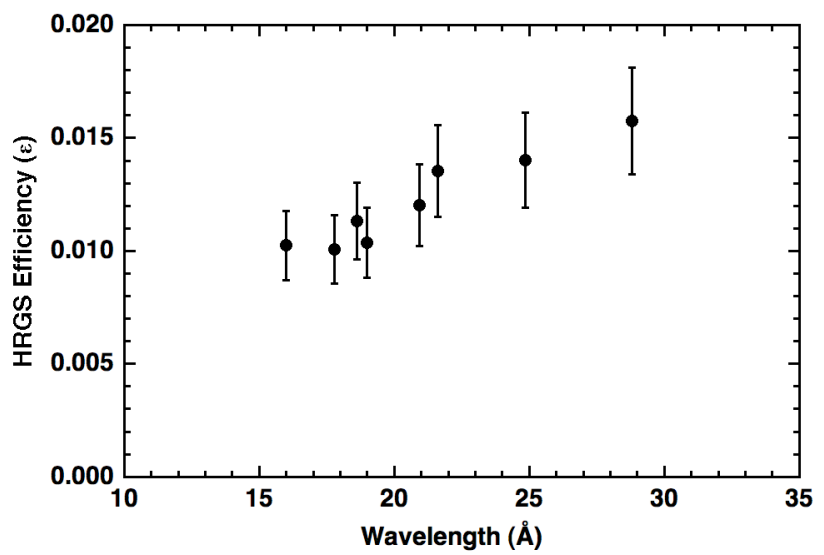

FIG. 2. The HRGS absolute efficiency, $\varepsilon$, defined here as grating reflectivity $\times$ quantum detection efficiency product as a function of wavelength for $15-30 \AA$. $\varepsilon$ is found to be $\sim 1-1.6 \%$. The grating reflectivity is expected to be very close to the above values.

In previous work to characterize the response of a backthinned CCD detector, typical values of dead layers of $10 \mathrm{~nm}$ of $\mathrm{SiO}_{2}$ as well as $20 \mathrm{~nm} \mathrm{Si}$ were determined. ${ }^{7}$ The active depth of the detector was also determined to be $10 \mu \mathrm{m}$. This would give a predicted detection efficiency that would gradually slope down from $97 \%$ at $15 \AA$ to about $90 \%$ at $30 \AA$. We can speculate that the values for the grating reflectivity are very close to the efficiency curve in Fig. 2 and so the reflectivity is $\sim 1-2 \%$. The future plan is to separately determine the CCD response as well as extend the calibration to a wider spectral range.

\section{CONCLUSIONS}

The calibration of a high resolution grating spectrometer has been reported using the FAST facility at LLNL and the EBIT calorimeter spectrometer. This allows the absolute calibration of the main components of the spectrometer namely the grating reflectivity and detector quantum efficiency where values of 1 $1.6 \%$ have been measured. The large area of the grating, allowing increased solid angles, together with the high spectral resolution capability make this a powerful instrument for high temperature plasmas.

\section{ACKNOWLEDGMENTS}

This work performed under the auspices of the U.S. Department of Energy by Lawrence Livermore National Laboratory under Contract DE-AC52-07NA27344.

${ }^{1}$ Plasma Diagnostic Techniques, ed.s R. H. Huddlestone and S. L. Leonard, (Academic Press, New York, 1965).

${ }^{2}$ H. R. Griem, Plasma Spectroscopy, (McGraw-Hill, New York, 1964).

${ }^{3}$ C. De Michelis and M. Mattioli, Nucl. Fus. 21(6), 677 (1981).

${ }^{4}$ J. D. Kilkenny, R. W. Lee, M. H. Key, and J. G. Lunney, Phys. Rev. A. 22(6), 2746 (1980).

${ }^{5}$ T. Kita, T. Harada, N. Nakano, and H. Kuroda, Appl. Opt. 22, 512 (1983).

${ }^{6}$ G. V. Brown, P. Beiersdorfer, J. Emig, M. Frankel, M. F. Gu, R. F. Heeter, E. Magee, D. B. Thorn, K. Widmann, R. L. Kelley, C. A. Kilbourne, and F. S. Porter, Rev. Sci. Instrum. 79, 10E309 (2008).

${ }^{7}$ Yuelin Li, G. D. Tsakiris, and R. Sigel, Rev. Sci. Instrum. 66(1), 80 (1995).

${ }^{8}$ P. Beiersdorfer, E. W. Magee, E. Träbert, H. Chen, J. K. Lepson, M. -F. Gu, and M. Schmidt, Rev. Sci. Instrum. 75, 3723 (2004).

${ }^{9}$ J. Dunn, E. W. Magee, R. Shepherd, H. Chen, S. B. Hansen, S. J. Moon, G. V. Brown, M. -F. Gu, P. Beiersdorfer, and M. A. Purvis, Rev. Sci. Instrum. 79, 10E314 (2008).

${ }^{10}$ P. Beiersdorfer, Can. J. Phys. 86, 1 - 10 (2008).

${ }^{11}$ G. V. Brown, P. Beiersdorfer, J. Dunn, E. Magee, J. Park, F. S. Porter, M. Schneider, E. Träbert, R. L. Kelley, and C. A. Kilbourne, "The Fusion and Astrophysics Data and Diagnostics Calibration Facility", in print in the Proceedings of the SPIE Astronomical Telescopes and Instrumentation 2010 Conference (2010).

${ }^{12}$ F. S. Porter, B. R. Beck, P. Beiersdorfer, K. R. Boyce, G. V. Brown, H. Chen, J. Gygax, S. M. Kahn, R. L. Kelley, C. A. Kilbourne, E. Magee, and D. B. Thorn, Can. J. Phys. 86, 231 (2008).

${ }^{13}$ R. L. Kelly, J. Phys. Chem. Ref. Data 16, 1 (1987).

${ }^{14}$ J. Dunn, P. Beiersdorfer, G.V. Brown and E.W. Magee, in X-ray Lasers 2008 , Proceedings of the $11^{\text {th }}$ International Conference on X-ray Lasers, 17 - 22 August 2008, Belfast, UK, Springer Proceedings in Physics Vol. 130, Ed.s C.L.S. Lewis, D. Riley, 461 - 7 (2009). 